\title{
ESTÁNDARES DE CALIDAD EN CIRUGÍA COLORRECTAL*
}

\author{
Dr. Guillermo Bannura C. ${ }^{1}$
}

1 Servicio y Departamento de Cirugía. Hospital Clínico San Borja Arriarán (HCSBA). Facultad de Medicina Universidad de Chile.

Santiago, Chile.

\begin{abstract}
Quality indicators for colorectal cancer management

The yield of colorectal cancer treatment significantly improves if a program with quality indicators is followed. These quality indicators are aimed to reduce surgical variability, improve results and reduce cost. Published experiences from developed countries demonstrate that, although quality indicators vary considerably, there is a consensus about their basal implementation. In the United States, a panel sponsored by the National Cancer Institute validated 92 indicators grouped in six domains that comprise all aspects of colorectal cancer patient management. The Mount Sinai Hospital of Ontario uses 15 such indicators and European hospitals use 50 indicators about diagnosis, treatment and follow up. The mortality rates for colorectal cancer are increasing in Chile, therefore the global results of its management should be assessed prospectively. We propose a quality indicator model that could be applied locally as a basis for a broader discussion about a model consented by all centers managing colorectal cancer, aiming to improve the efficiency in its management.

Key words: Colorectal cancer, quality indicators, surgery.
\end{abstract}

\section{Resumen}

Estudios publicados en el extranjero muestran una mejoría significativa del manejo del cáncer colorrectal (CCR) luego de la implementación de un Programa basado en algunos estándares de calidad cuyo objetivo principal es disminuir la variabilidad de la práctica quirúrgica, mejorar los resultados y contener los costos asociados. Se revisa algunas experiencias publicadas en el mundo desarrollado, comprobando que, aunque los esquemas aplicados varían en cantidad y en calidad, existe un consenso sobre un nivel basal de indicadores necesarios para medir los resultados, hacer las correcciones necesarias y comparar en forma longitudinal en el tiempo, los avances en el manejo global del CCR. En USA un panel patrocinado por el NCI validó 92 indicadores en 6 dominios que abordan todos los aspectos relacionados con el manejo del paciente con un CCR, mientras que la Universidad de Toronto-Hospital Mount Sinaí (Ontario) utiliza 15 indicadores con estos fines. Estudios en Europa muestran modelos parecidos con alrededor de 50 indicadores que abarcan la etapa de diagnóstico, tratamiento y seguimiento de esta patología. La tasa de mortalidad por CCR en Chile se ha elevado en los últimos 20 años, lo que nos obliga a evaluar lo que estamos haciendo en el manejo global del CCR. No existiendo un modelo, se propone uno potencialmente aplicable en los centros nacionales que se

*Recibido el 29 de junio de 2013 y aceptado para publicación el 2 de agosto de 2013.

El autor no refiere conflictos de interés.

Correspondencia: Dr. Guillermo Bannura C.

Las Limas 1622, Santiago, Chile.

gbannura@gtdmail.com 
dedican al manejo del CCR, como una base para una discusión amplia, cuyo objetivo es la estandarización del cuidado global de esta neoplasia. El cotejar nuestra práctica quirúrgica con los estándares consensuados se traduce en la implementación de nuevas técnicas de estudio y tratamiento y, en definitiva, en mejores resultados finales con un claro beneficio de nuestros pacientes.

Palabras clave: Cáncer colorrectal, indicadores de calidad.

\section{Introducción}

En los últimos años una serie de organismos estatales, sociedades científicas y entidades específicamente nombradas por la autoridad en Salud en el mundo desarrollado, han destacado la necesidad creciente de disminuir la variabilidad de la práctica quirúrgica, como una forma de mejorar los resultados y contener los costos asociados a la cirugía moderna $^{1}$. En USA, agencias como el Centers for Medicare and Medicaid Services (CMS), la Joint Comission on Accreditation of Healthcare Organizations (JCAHO) y la National Quality Forum (NQF) han desarrollado formas de medir la calidad del cuidado, especialmente en el manejo de la patología neoplásica ${ }^{2}$. Un estándar se entiende como el grado de adherencia a una guía de práctica clínica (GPC), protocolo o recomendación basada en la mejor evidencia médica disponible, consistente con el conocimiento médico actualizado. Ello implica cotejar los resultados en el manejo de una patología mediante indicadores sensibles, válidos y relevantes, lo que inevitablemente conduce a la comparación con centros de complejidad similar, con el fin de corregir, incorporar nuevas tecnologías y mejorar los resultados dentro de la propia Institución en una evaluación longitudinal a través del tiempo.

El cáncer colorrectal (CCR), por su frecuencia en el mundo desarrollado, no ha estado ajeno a este proceso y son numerosas las agencias en USA que han diseñado indicadores de calidad que representan el nivel básico del manejo del CCR. Entre ellas podemos mencionar la American Society of Clinical Oncologist (National Comprehensive Cancer Network $(\mathrm{NCCN})^{3}$, la American Society of Colon and Rectal Surgeons (ASCRS) ${ }^{4}$ y el National Cancer Institute $(\mathrm{NCI})^{5}$. La necesidad de regulación de la práctica quirúrgica en el manejo del CCR queda de manifiesto al observar las estadísticas del SEER ${ }^{6}$, según la cual sólo en el 30\% de los pacientes operados por cáncer de colon cumplen las recomendaciones del NCI, el 33\% de las piezas resecadas tienen un recuento ganglionar igual o superior a 12 linfonodos y alrededor del $30 \%$ de los pacientes en estadio III reciben la quimioterapia aconsejada. De este modo, en un panel de consenso patrocinado por el NCI y luego de una revisión sistemática de la literatura, los participantes examinaron 142 potenciales indicadores de calidad en 6 dominios, validando 92 que abordan todos los aspectos relacionados con el manejo del paciente con un CCR, los cuales a veces difieren de las GPC desarrolladas por la NCCN, la ASCRS y del $\mathrm{NCI}^{7}$. El esquema planteado es muy detallado y completo e incluye indicadores que son muy generales y que pueden ser muy obvios para el cirujano especialista (ej. si el paciente va a ser operado de un cáncer de recto bajo, debe colocarse en la mesa en posición de litotomía-Trendelemburg; debe identificarse el uréter del lado intervenido; si ocurre una lesión iatrogénica, ésta debe ser documentada en el protocolo operatorio, etc.), lo que consume un tiempo valioso, por lo cual creemos que en una primera fase, al menos, debemos enfocarnos en los indicadores de calidad más relevantes.

La ventaja de los indicadores de calidad es que son medibles, y que no sólo incluye indicadores de estructura (poco modificables), de resultados (algo que ya ocurrió) sino que también mide indicadores de procesos (modificables). Esto permite que las agencias de acreditación, así como los pacientes y las agencias aseguradoras, tengan información precisa de las competencias de los cirujanos (ej: experiencia en 20 casos de resección laparoscópica del colon durante su residencia), de las prácticas habituales (ej: estudio preoperatorio que incluya CEA) y los resultados específicos del centro analizado (indicadores de resultados en mortalidad, recidiva, reoperaciones no programadas, etc.). Esto tiene implicancias económicas (ej: compañías aseguradoras que no pagan por estudios histopatológicos incompletos de acuerdo al estándar) y legales que son obvias y de gran impacto global.

\section{Algunas experiencias publicadas}

En otros países, centros de reconocida trayectoria han elaborado indicadores de calidad para el CCR basado en su propia experiencia, como el Hospital Mount Sinai-Universidad de Toronto, Canadá, bajo el auspicio del Departament of Health Policy ${ }^{8}$, que incluyen la evaluación preoperatoria, el período peri-operatorio, el estudio de la pieza quirúrgica y el seguimiento, lo que se expresa como la proporción de pacientes operados en que se cumplió con el estándar. Ejemplo: Proporción de pacientes que tenían un estudio preoperatorio por imágenes del Hígado con ultrasonografía (ECO), tomografía computada 
(TC) o resonancia magnética (RM). Particularmente de importancia, se incorporan los resultados finales de esta práctica en términos de dehiscencia anastomótica, mortalidad operatoria, recurrencia local y sobrevida a largo plazo. El período de 10 años estudiado les permite, además, hacer un análisis comparativo tanto de los resultados de la cirugía del CCR como del cumplimiento de los estándares de calidad elegidos. En el año 2005, un panel multidisciplinario propuso 15 indicadores para el CCR que son, en su mayoría, susceptibles de incorporarse como guía local (Tabla 1), luego de lo cual se aprecian avances significativos en cuanto al cumplimiento de las recomendaciones y una estandarización de los procedimientos.

En la región de Aquitania (Francia), que tiene una cobertura de 3 millones de habitantes y con el fin de conocer la realidad local, se investigó la adherencia a las GPC en una cohorte de 1.206 pacientes operados en 53 hospitales durante un período de 1 año, tras la implementación de GPC para el CCR ${ }^{9}$. El $26 \%$ de los pacientes con cáncer con estadio II y $71 \%$ en estadio III recibieron quimioterapia adyuvante. De los pacientes con un tumor de recto etapificado por ultrasonido como T3T4, el 84\% recibieron radioterapia preoperatoria. La recomendación de una cosecha ganglionar $>12$ linfonodos se cumple en pacientes de mejor status perfomance, hospitales de alto volumen y tumores más avanzados. La quimioterapia adyuvante se asocia con pacientes jóvenes, estadios avanzados, urgencias y el tipo de institución (público/privada). Los autores concluyen que es necesario focalizar en estos grupos la implementación de los estándares de calidad aceptados por el grupo de expertos.

En Alemania, especialistas de la Universidad de Heidelberg con el apoyo del Instituto de Salud de Alemania, validaron 52 indicadores de calidad para el manejo integral del $\mathrm{CCR}^{10}$. Luego de una revisión exhaustiva de la literatura, escogieron 11 indicadores que cubren los procedimientos diagnósticos, 28 indicadores del manejo terapéutico y 6 indicadores en la etapa de seguimiento. En la fase diagnóstica destacan la existencia de un equipo multidisciplinario en el estudio y la toma de decisiones con experiencia en enfermedad metastásica, la calidad y contenido del informe de la colonoscopía preoperatoria, la evaluación con rectoscopía rígida en los casos de cáncer del recto, la aplicación del TNM clínico, los estudios por imagen y la evaluación de la función sexual y urinaria en los pacientes con tumores de recto. En la fase preparatoria, destacan la educación a paciente por una estoma-terapeuta y la marcación del estoma, cuando corresponda. En la fase terapéutica señalan la neoadyuvancia con radioquimioterapia para el cáncer de recto y la forma y dosificación

\section{Tabla 1. Modelo de Ontario: 15 indicadores de calidad en el manejo del CCR}

1. Proporción de pacientes (PDP) de CCR detectados por screening

2. PDP con estudio del colon (colonoscopía o enema baritada) 3 meses antes de la cirugía o 6 meses después de ésta

3. PDP con estudio preoperatorio del hígado con USER, TC o RM

4. PDP con cáncer de recto con estudio de la pelvis con TC o RM

5. PDP con cáncer de recto evaluado por un radioterapeuta antes de la cirugía

6. PDP que fue evaluado por un oncólogo médico en el preoperatorio

7. PDP operados por cáncer de recto cuyo protocolo operatorio menciona la disección de mesorrecto, localización y extensión del tumor, grado de preservación neural y extensión de la linfadenectomía

8. PDP operados con estudio histopatológico que incluye el número de linfonodos examinados y el número de linfonodos positivos

9. PDP operados cuyo estudio histopatológico señala detalles del margen radial (positivo $<1 \mathrm{~mm}$ ) y distal (positivo $<1 \mathrm{~cm})$

10. Proporción de informes patológicos que señalan el margen distal y radial en forma cuantitativa

11. PDP operados por cáncer de recto que evolucionan con dehiscencia anastomótica

12. Mortalidad intrahospitalaria (dentro de 30 días)

13. Tasa de recidiva local en CCR

14. Sobrevida global y ajustada a 5 años

15. PDP con cáncer de colon con una colonoscopía dentro de 1 año de seguimiento luego de la cirugía

PDP: proporción de pacientes. TC: tomografía computada RM: resonancia magnética. USER: ultrasonografía endorrectal. 
de la misma según los estándares de la Sociedad Germana de Radioterapia. En el intraoperatorio, se releva la importancia de la antibioticoprofilaxis, la resección en block, la resección total del mesorrecto, la medición de la tasa de dehiscencia anastomótica en cirugía electiva, el porcentaje de reoperaciones y la mortalidad operatoria. El estudio de la pieza quirúrgica incluye el recuento ganglionar de al menos 12 linfonodos, el porcentaje de pacientes con R0, el porcentaje de pT1 y de pacientes en estadio IV, el estudio del margen radial en cáncer de recto y la adherencia a las pautas de la Sociedad Alemana de Patología. En el seguimiento se destaca la quimioterapia adyuvante y el intervalo de la misma en estadios III, la evaluación de la función sexual y urinaria, la instrucción en el manejo de la ostomía y en el seguimiento la colonoscopía dentro de 6 meses para los pacientes con una colonoscopía incompleta. Desde la perspectiva de los pacientes, incorpora indicadores como la toma de decisiones con el paciente (consentimiento informado), la oportunidad del paciente de preguntar en un espacio y tiempo protegido, el apoyo psicológico y la calidad de vida posterior a la cirugía. Finalmente, los autores incluyen indicadores biomédicos como la sobrevida a 5 años, la recidiva local y la medición de la calidad de vida con un instrumento específico.

\section{Situación en Chile}

No hemos encontrado publicaciones nacionales específicas sobre los indicadores de calidad en el tratamiento del CCR en nuestro país, aunque es evidente que gran parte de los estándares señalados más arriba se cumplen en la mayoría de los centros que habitualmente realizan este tipo de cirugía, tanto de Santiago como en provincia. De hecho, varias publicaciones nacionales señalan expresamente el tipo de estudio realizado a sus pacientes, la toma de decisiones en un equipo multidisciplinario (Comité Oncológico), la neoadyuvancia en cáncer de recto y el tipo de cirugía realizada, el protocolo del estudio histopatológico de la pieza quirúrgica, así como los resultados inmediatos y alejados en términos de recidiva y sobrevivencia ${ }^{11-16}$. En la Tabla 2 se muestra los indicadores utilizados en el HCSBA,

Tabla 2. Indicadores de calidad en el manejo del CCR* en el HCSBA y tasa de cumplimiento

\begin{tabular}{|c|c|}
\hline Indicador & $\%$ \\
\hline Estudio preoperatorio con colonoscopía y/o enema $\mathrm{Rx}$ & 95 \\
\hline Estudio con TC y/o RM y/o USER & 90 \\
\hline Evaluación por radioterapeuta y oncólogo preop. en cáncer de recto bajo & 90 \\
\hline Evaluación preoperatoria en comité & 100 \\
\hline Cirugía laparoscópica (intención) & 70 \\
\hline Protocolo operatorio que señala límites de la resección, resección en block, márgenes distal y radial con esquema & 95 \\
\hline Informe patología detallado (margen radial, distal, recuento LN y LN positivos, permeación linfática y perineural & 100 \\
\hline CEA preoperatorio & 90 \\
\hline Neoadyuvancia en cáncer de recto bajo localmente avanzado & 80 \\
\hline Dehiscencia anastomótica global & 3,8 \\
\hline Dehiscencia en anastomosis colorrectal baja & 12 \\
\hline Reoperaciones & 5,7 \\
\hline Mortalidad operatoria (90 días) cáncer de colon (cirugía radical) & 0,9 \\
\hline Mortalidad operatoria (90 días) cáncer de recto (cirugía radical) & 1,4 \\
\hline Tasa de recidiva local en cáncer de recto bajo (cirugía radical) & 12 \\
\hline Sobrevida global a 5 años de cáncer de colon (curativos) & 82 \\
\hline Sobrevida global a 5 años de cáncer de recto (curativos) & 70 \\
\hline Cirujano tratante certificado en la especialidad & 100 \\
\hline Seguimiento selectivo con CEA postoperatorio & 90 \\
\hline
\end{tabular}

LN: linfonodos. CEA: antígeno carcinoembrionario. TC: tomografía computada. RM: resonancia magnética. USER: ultrasonografía endorrectal. Cirugía electiva por CCR. 
hospital público docente como un insumo, para la discusión y enriquecimiento del tema, lo que podría ser abordado en el seno de la Sociedad Chilena de Coloproctología. Entre las múltiples carencias cabe señalar la ausencia de una estoma-terapeuta con dedicación exclusiva como lo señala el estándar, con la función de atender en forma transversal (ambulatorios y hospitalizados, adultos y niños) a todos los pacientes que lo requieran, con la debida educación y preparación desde antes del procedimiento, cuando ello corresponda. Por otra parte, quedan pendientes muchos aspectos del cuidado del CCR que requieren una definición local, de acuerdo al nivel de complejidad y de los recursos de la Institución. La certificación de los médicos especialistas en la sub-especialidad de la Coloproctología, los servicios de apoyo en radiología, laboratorio y anatomía patológica de la Institución y los volúmenes quirúrgicos en un centro específico, son aspectos relevantes en la toma de decisiones (desde el año 2009 en Francia, no se autoriza el tratamiento de un paciente con un cáncer digestivo si el número de casos anuales de ese centro es menor a 30$)^{9}$. Temas más específicos como la necesidad de contar con resonancia magnética en la evaluación preoperatoria del cáncer de recto avanzado (lo que implica incluir este recurso en la canasta Auge del cáncer de recto), las indicaciones de la USER para tumores móviles y pequeños, las indicaciones de quimioterapia en estadios II de cáncer de colon (definir pacientes de alto riesgo: operados por obstrucción, T4, CEA preoperatorio elevado, etc.), el rol de la quimioterapia luego de neoadyuvancia más cirugía radical por cáncer de recto bajo, el protocolo del estudio histopatológico de la pieza resecada (cosecha ganglionar, margen radial, depósitos tumorales, etc.), seguimiento con CEA selectivo, son algunos de los aspectos debatibles que requieren de una discusión multidisciplinaria y de un consenso a nivel nacional.

Un tema pendiente en nuestra realidad es el indicador porcentaje de pacientes intervenidos por un CCR que se detectaron en la etapa pre-sintomática, es decir, mediante un screening efectuado en población aparentemente sana. En Chile se desconoce la prevalencia del CCR, existe una seria deficiencia en recursos de especialista y endoscopías en un escenario en que la tasa de mortalidad por CCR se eleva desde 4,9 por 100.000 en el año 1990 a 7,2 por 100.000 en el año 2000 y a 10,2 en el 2008 (DEIS 2010). Actualmente, existe en Chile un programa piloto de detección precoz del CCR, resultado de un convenio de cooperación entre el Ministerio de Salud, Tokio and Dental University y Clínica Las Condes, que busca examinar mediante una colonoscopía a 16.000 personas mayores de 50 años cuyo Fecatest sea positivo. Los resultados de este programa, que incluye a 5 regiones del país (Santiago Centro-HCSBA ${ }^{17}$, Punta Arenas, Antofagasta, La Serena y Temuco), pueden orientar en forma consistente las políticas públicas de control del CCR el cual, a juzgar por las tasas de mortalidad, se ha duplicado en las últimas 2 décadas. Esperamos que al implementar un programa de diagnóstico precoz y prevención secundaria de CCR en el HCSBA, podamos cumplir en un futuro no lejano con este indicador y subir la tasa de pacientes intervenidos en los estadios iniciales de la enfermedad.

\section{Referencias}

1. Malin JL, Asch SM, Kerr EA, McGlynn EA. Evaluating the quality of cancer care: development of cancer quality indicators for a global quality assessment tool. Cancer 2000;88:701-707.

2. Centers for Medicare\&Medicaid Services. Available at http://www.cms.hhs.gov.

3. Desch CE, McNiff KK, Schneider EC, Schrag D, McClure J, et al. American Society of Clinical Oncology/ National Comprehensive Cancer Network Quality Measures. J Clin Oncol. 2008;26:3631-7.

4. Otchy D, Hyman NH, Simmang C, Anthony T, Buie WD, Cataldo P, et al. Practice parameters for colon cancer. Dis Colon Rectum 2004;47:1269-84.

5. Nelson H, Petrelli N, Carlin A, Couture J, Fleshman J, Guillem J, et al. Guidelines 2000 for colon and rectal cancer surgery. J Natl Cancer Inst. 2001;93:583-96.

6. Howlader N, Noone AM, Krapcho M, Neyman N, Aminou R, Waldron W, et al. (eds). SEER Cancer Statistics Review, 1975-2008, National Cancer Institute. Bethesda, MD,http://seer.cancer.gov/csr/1975_2008.

7. McGory ML, Skekelle PG, Ko CY. Development of quality indicators for patients undergoing colorectal cancer surgery. J Natl Cancer Inst. 2006;98:1623-33.

8. Vergara-Fernandez O, Swallow CJ, Victor JC, O'Connor BI, Gryphe R, MacRae HM et al. Assessing outcomes following surgery for colorectal cancer using quality of care indicators. J Can Chir. 2010;53:232-40.

9. Mathoulin-Pélissier S, Bécouarn Y, Belleannée G, Pinon E, Jaffré A, Coureau G, et al. Quality indicators for colorectal cancer surgery and care according to patient, tumor, and hospital-related factors. BMC Cancer 2012;12:297.

10. Ludt S, Urban E, Eckardt J, Wache S, Broge B, Kauffmann-Kolle P, et al. Evaluating the quality of colorectal cancer care across the interface of heathcare sectors. PLoS ONE 8 (5): e60947. Doi: 10.1371/journal. pone.0060947.

11. Espinoza D, Molina ME, Bellolio F, Gellona J, Bustos M, Zúñiga A. Respuesta patológica completa en pacientes sometidos a neoadyuvancia en cáncer de recto. Rev Chil Cir. 2013;65:333-7.

12. Bannura G, Vargas C, Barrera A, Melo C, Illanes F. 
Valor pronóstico de la respuesta patológica a la radioquimioterapia preoperatoria en el cáncer de recto bajo localmente avanzado. Rev Chil Cir. 2013;65:236-41.

13. Bannura G, Vargas C, Barrera A, Melo C, Contreras J. Índice ganglionar y número de linfonodos metastásicos como factores pronósticos en cáncer de colon. Rev Chil Cir. 2011;63:485-92.

14. Gellona J, Urrejola G, Quezada F, Miguieles R, Molina ME, Bellolio F, y cols. Resección laparoscópica en cáncer de recto etapa III ¿mejor de lo que esperábamos? Rev Chil Cir. 2013;65:35-40.

15. Bannura G, Cumsille MA, Barrera A, Contreras J, Melo
C, Soto D. Resultados del tratamiento quirúrgico del cáncer de colon. Análisis de 439 pacientes. Rev Chil Cir. 2010;62:491-6.

16. Bannura G, Del Castillo C, Cumsille MA, Barrera A, Contreras J, Soto D, y cols. Resultados de la quimioterapia adyuvante en cáncer de colon. Rev Chil Cir. 2008;60:403-9.

17. Mejoramiento de la Unidad de Endoscopía digestiva en Hospital Clínico San Borja Arriarán de Santiago para el diagnóstico precoz y la prevención secundaria de cáncer colorrectal. Marzo 2013. MINSAL. COD BIP 30122324-0. 